\title{
The impact of maternal singing on glucocorticoids, cytokines, anxiety, affect and mother-infant bonding
}

\author{
Daisy Fancourt ${ }^{\mathrm{a} \mathrm{b}^{*}}$ and Rosie Perkins ${ }^{\mathrm{b}, \mathrm{c}}$ \\ a Research Department of Behavioural Science and Health, University College London \\ b Faculty of Medicine, Imperial College London \\ c Centre for Performance Science, Royal College of Music
}

Background: There has been growing research on the impact of maternal singing on infants, with data showing both reductions in behavioural anxiety and improvements in physiological measures. However, to date there has been little quantitative exploration of the impact of infant-directed singing on mothers.

Methods: Two studies were conducted: a within-subject cross-over design with 43 mothers (aged 28-45) with babies aged 3-14 months old, and a randomised controlled trial with 101 mothers (aged 22-47) with babies aged 1-9 months old. In both groups, sessions of mother-infant singing involving 10-12 mothers in a group were compared with a control condition involving chatting and playing with the babies. Participants completed psychological questionnaires (measuring affect, anxiety and mother-infant bonding) and gave saliva samples pre- and post-each activity to test for cortisol, cortisone, DHEA and a range of cytokines (IL-1 $\beta$, IL6, IL10 and IFN-Y).

Results: Singing led to significantly greater increases in mother-infant bonding and positive affect than the control condition alongside significantly greater reductions in negative affect and anxiety. It also led to significantly greater reductions in cortisol, but not cortisone or DHEA. Singing also led to significantly greater decreases in IL-1 $\beta$ and IFN-y but not IL-6 or IL-10.

Conclusions: Overall, however, this study demonstrates for the first time the psychobiological impact of infantdirected singing on mothers. It sheds light on our evolutionary understanding of the role of singing for mothers and their infants. Further research remains to be undertaken to explore the longer-term psychobiological effects of mother-infant singing on maternal health and wellbeing. 